\title{
ALIANZAS ENTRE EMPRESAS LUCRATIVAS Y ORGANIZACIONES SIN FINES DE LUCRO (OSFL). ESTUDIOS DE CASOS EN CHILE ${ }^{1}$
}

\author{
Alliances between Private Firms and Nonprofit Organizations. \\ Studies of cases in Chili \\ Darío Rodríguez $\left(^{*}\right)$ \\ Rodrigo Flores $\left({ }^{* *}\right)$ \\ Paula Miranda $\left({ }^{* * *}\right)$
}

\section{RESUMEN}

Este artículo pretende estudiar las alianzas de cooperación de OSFL chilenas con organizaciones donantes, describiendo las principales características de dichas alianzas y su dinámica evolutiva. Presenta un modelo conceptual que permite conocer las relaciones de colaboración existentes entre OSFL y organizaciones

(*) Facultad de Ingeniería, Universidad Diego Portales. Santiago, Chile. Correo electrónico: dariorodrigue@gmail.com

$\left.{ }^{(*}\right)$ Facultad de Ciencias Sociales, Pontificia Universidad Católica de Chile. Santiago, Chile. Correo electrónico: rfloresu@uc.cl

${ }^{(* *)}$ Facultad de Ciencias Sociales, Pontificia Universidad Católica de Chile. Santiago, Chile. Correo electrónico: pmirands@uc.cl

${ }^{1}$ Este artículo da cuenta de los principales hallazgos de la primera fase de una investigación financiada por Fondo Nacional de Desarrollo Científico y Tecnológico (FONDECYT) Nº 1120492.

Artículo recibido el 25 de enero de 2012. Aceptado el 18 de abril de 2013. 
donantes, donde el producto de tal relación es la generación de valor social y económico. Los resultados indican que estas alianzas surgen a partir de contactos pre-existentes que aportan la confianza necesaria; que falta planificar a largo plazo el futuro de las alianzas; que ambas aliadas se benefician y que es preciso profesionalizar la gestión de una alianza.

\title{
Palabras clave:
}

Relaciones de colaboración, empresas, Organizaciones Sin Fines de Lucro.

\begin{abstract}
This paper aims to explore cooperation alliances between Chilean Nonprofit organizations and donating private firms. It describes the main features of those alliances, and their developmental dynamics. It offers a conceptual model which permits the understanding of collaborative relations between Nonprofits and their donor firms, aimed to generate both social and economic value. Research results show that these alliances emerge based on pre-existing social contacts that bring the required social trust; there is not enough long term planning of the alliance's future; both related organizations benefit; and it is essential to professionalize an alliance's management.
\end{abstract}

Keywords:

Inter-organizational collaborative relationships, companies, NGO.

\section{INTRODUCCIÓN}

Las Organizaciones Sin Fines de Lucro (OSFL) son actores activos en la economía chilena. En 2006 se estimó que el sector empleaba de forma remunerada o voluntaria sobre las 303 mil personas en Jornada Completa Equivalente y que su contribución al PIB chileno era de aproximadamente un 1,5\%, representando un 2,6\% del empleo remunerado (Hopkins, 2006), duplicando en importancia a sectores sin fines de lucro de países como Brasil y Colombia (Radrigán, Dávila y Penaglia, 2012). Aunque se cree que se financian en gran medida a través de proyectos sustentados internacionalmente o estatalmente subsidiados, no se conoce 
con exactitud cómo logran mantenerse en el tiempo y apenas se ha investigado cómo logran elaborar y mantener vínculos con otras clases de organizaciones.

En Chile, las OSFL se mueven en entramados sociales de alta informalidad. En una estimación al año 2002, la distribución porcentual en nuestro país de los ingresos de las OSFL se estructuraba de la siguiente forma: Estado (17\%) - Filantropía (16\%) - Cuotas/venta de servicios (67\%) (Ríos, 2002). Es importante destacar que, en comparación con el resto de América Latina, Chile muestra una mayor proporción de aporte filantrópico y estatal (Mangili, 2005).

El presente artículo pretende dar cuenta de las alianzas de colaboración entre OSFL chilenas con organizaciones donantes, describir las principales características de dichas alianzas y observar la dinámica evolutiva de estas relaciones de cooperación. Hace algunos años en nuestro país, un estudio hizo notar que las relaciones de colaboración efectivas entre OSFL y empresas con afán de lucro se construyen sobre estrechos nexos interpersonales previos de amistad o parentesco. Por esta misma razón, dichas alianzas son altamente personalizadas, dependen mucho de la atención y energía que les brinden los líderes vinculados y no se proyectan más allá del corto plazo (Koljatic y Silva, 2002).

Este estudio pretende elaborar un modelo conceptual que permita conocer las relaciones de colaboración existentes entre OSFL orientadas a servicios humanos y organizaciones donantes, donde el producto de tal relación es la generación de valor social y económico. Para ello se enfoca en lo que los directivos o representantes de OSFL orientadas a servicios humanos informan sobre las características de su alianza.

\section{TRES SECTORES}

Las organizaciones se constituyen en sistemas característicos de la sociedad compleja, relacionándose con ella de tal manera que algunos se forman a partir de los sistemas de funciones de la sociedad, asumiendo su correspondiente primado funcional, buscando soluciones específicas a los problemas que deben afrontar. De esta manera, la economía es asumida como temática comunicacional propia de las empresas, la educación en los establecimientos educacionales, la salud 
en los hospitales, la política en distintos partidos, la justicia en los juzgados, el arte en museos y galerías y las religiones en las iglesias y sus particularidades, manteniéndose así la condición de que cada uno se vuelve a diferenciar en otros tantos sistemas organizacionales y así sucesivamente (Rodríguez, 2001; Flores y Donoso, 2012). Sin embargo, dado que los problemas de la sociedad son siempre complejos y difusos, las organizaciones necesitan especificar sus objetivos para estar en condiciones de alcanzarlos, por lo cual no logran abarcar la totalidad del problema y, por lo mismo, siempre son insuficientes (Luhmann, 2007, 2011).

Una de las formas de diferenciación más difundidas divide las áreas organizacionales de acción en tres grandes ámbitos: público, privado y tercer sector. De manera muy esquemática y simplificada, se puede decir que esta forma de diferenciación de las organizaciones se relaciona con la diferenciación de la sociedad mundial en subsistemas funcionales, en que el subsistema político se ha estructurado bajo la forma de estados nacionales encargados de la regulación de las relaciones políticas de un cierto territorio, bajo una forma predominantemente democrática (Luhmann, 2007). Las organizaciones públicas forman parte del aparato estatal o dependen de él y se encargan de la solución de los problemas que les sean delegados por este aparato. El subsistema económico ha globalizado las actividades económicas según un modelo de mercado y las organizaciones dedicadas a ello persiguen fines de lucro, acordes con dicho modelo. Dado que, como se ha dicho, los problemas de la sociedad son complejos, difusos y no admiten especificaciones, las organizaciones públicas y privadas dejan parte importante de dichos problemas sin considerar. Esta es la razón por la cual emerge un tercer sector, constituido por Organizaciones No Gubernamentales (ONG), que buscan enfrentar los problemas insuficientemente tratados por las organizaciones públicas gubernamentales, y Organizaciones Sin Fines de Lucro (OSFL), que velan por problemas dejados de lado por las organizaciones privadas con fines de lucro. Aunque se pueda vincular las organizaciones del tercer sector con problemas no atendidos por las organizaciones públicas o privadas, en definitiva todas ellas se caracterizan por no ser dependientes del Estado ni perseguir fines de lucro y por eso se las puede denominar indistintamente ONG u OSFL. Otra explicación de la diferencia entre las Organizaciones Sin Fines de Lucro y las No Gubernamentales indica que estas últimas son organizaciones sin fines de lucro del área del desarrollo internacional, en tanto las organizaciones sin fines de lucro propiamente tales operarían en países 
industrializados. Si bien esta segunda explicación no nos parece adecuada, porque reduce la diferencia al área de operaciones y no permite entender la razón de la distinción conceptual, finalmente también concluye que se las puede llamar de ambas formas (Brinkerhoff, 2002: 19).

La diferenciación de las actividades organizacionales en tres sectores reduce la complejidad característica de los problemas de la sociedad subdividiéndolos en aspectos a ser resueltos por el subsistema político, aspectos a ser resueltos por el subsistema económico y aspectos fuera de la competencia de estos dos subsistemas y que, pudiendo ser considerados por otros diversos subsistemas, parece conveniente agruparlos en un solo gran tercer sector. Esta diferenciación imprime a las organizaciones de los tres sectores la racionalidad predominante en el subsistema en que operan. De esta forma, las organizaciones públicas siguen la racionalidad político-administrativa propia del sistema político. En este sistema, las actividades organizacionales se abocan, por una parte, al juego político de buscar y encontrar legitimidad para la toma de decisiones y, por otra, a tomar decisiones administrativas que descansan en la legitimidad lograda en el juego político. Los partidos políticos son organizaciones dedicadas al juego de la búsqueda de legitimidad y los servicios públicos se encargan de las decisiones administrativas, cuya legitimidad no necesitan buscar porque está políticamente garantizada. Las organizaciones privadas, por su parte, siguen la racionalidad económica del mercado. La coordinación de las actividades económicas no se realiza por medio del juego político de la búsqueda de legitimidad ni por la lógica administrativa de la administración de la legitimidad. En el mercado, la coordinación se lleva a cabo mediante el afán de lucro que lleva a las organizaciones a competir buscando aumentar su parte de la utilidad que se pueda generar. Las organizaciones del tercer sector, no teniendo un subsistema propio que pueda impartirles su racionalidad, se definen negativamente y, por eso, son no gubernamentales y sin fines de lucro. La diferenciación de los tres sectores solamente persigue reducir la complejidad de los grandes problemas de la sociedad para enfrentarlos mediante la especialización de las organizaciones destinadas a ello. No obstante, esta diferenciación aparentemente inocua trae consigo la dificultad de que las racionalidades asumidas por las organizaciones de esos tres sectores son muy diferentes y no les resulta fácil dialogar ni comprenderse entre sí. Naturalmente, las barreras son mayores con las organizaciones cuya racionalidad se define negativamente con respecto a las 
racionalidades de los otros dos sectores. Y, para complicar aún más las cosas, los problemas de la sociedad siguen siendo amplios y difusos y no se dejan encasillar en los sectores definidos por la diferenciación.

Esta brevemente reseñada situación condujo a que, durante mucho tiempo, fuera prácticamente imposible la colaboración e incluso el entendimiento entre organizaciones públicas, privadas y del tercer sector. Los conflictos eran frecuentes y el diálogo era de sordos. Toda acción de una organización pública encontraba la crítica cuando no el repudio de las organizaciones privadas y el afán de lucro de las organizaciones privadas, era señalado con el dedo por las organizaciones públicas. Tampoco era fácil la comprensión entre las organizaciones de los dos primeros sectores y las del tercer sector. Las organizaciones públicas eran renuentes a compartir sus recursos y sus problemas con organizaciones que no tenían su misma lógica administrativa basada en la legitimidad política. Las organizaciones privadas miraban con recelo a estas organizaciones sin finalidad lucrativa en un mundo donde "todo" era entendido en términos del lucro. Las organizaciones del tercer sector tampoco lograban comprender a ninguna de las otras. La racionalidad administrativa les parecía inoperante, la búsqueda de legitimidad política les producía rechazo y la escasez de recursos provenientes del Estado les generaba sospechas respecto a contener intenciones diferentes a la solución de problemas sociales. El afán de lucro las disgustaba y exhibían ostentosamente su calidad de sin fines de lucro, pretendiendo demostrar alguna mayor altura moral ante quienes tenían el lucro como objetivo.

\section{RELACIONES DE COLABORACIÓN ENTRE ORGANIZACIONES}

Las condiciones de incomprensión que hemos descrito hacían complejo intentar colaborar entre organizaciones de diversos sectores. Aunque en la relación entre empresas privadas y OSFL, la forma filantrópica se presenta como la más frecuente (Austin, 2003: 44), desde fines del siglo XX ha aumentado la demanda, hecha a las OSFL por parte de sus donantes, de que mejoren su eficiencia en el uso de los recursos que les son otorgados (Koljatic y Silva, 2002: 5). La declinación del Estado de Bienestar ha implicado que las organizaciones del sector público restrinjan sus programas sociales, esperando que los servicios que ellas ofrecían sean asumidos por el tercer sector (Foster y Meinhard, 2002: 549-550). 
Las organizaciones lucrativas también han cambiado su perspectiva, acercándose cada vez más a establecer relaciones de colaboración con organizaciones no lucrativas.

La colaboración entre empresas con afán de lucro y OSFL puede ser definida como acuerdos voluntarios y cooperativos cuyo objetivo es mejorar la posición competitiva y el desempeño de empresas y OSFL (Austin y Seitanidi, 2012; Bouwen y Taillieu, 2004; Jamali y Keshishian, 2009; Koljatic y Silva, 2008; Kolk et al., 2008; Seitanidi, 2010; Wohlstetter et al., 2005). Envuelven una cuidadosa consideración de la elección del socio (Seitanidi et al., 2011), una misión interconectada (Austin, 2000; Vangen y Huxham, 2011), alineamiento de valores (Samii et al., 2002), una clara asignación de administración de responsabilidad en las áreas de competencia de ambas organizaciones (Austin, 2000; Austin, 2003), compromiso de recursos, comunicación abierta y constructiva.

La mayoría de la literatura que aborda las alianzas se ha centrado en delinear la naturaleza de la colaboración y distinguir entre distintos niveles y grados de intensidad de esta cooperación. Los autores coinciden en que se trata de un continuo incremental que va desde expresiones puramente filantrópicas hasta aquellas que asumen formas basadas en el involucramiento y rendición de cuentas entre ambas organizaciones (Austin, 2000, Austin et al, 2006; Bowen, Newenham y Herremans, 2010; Brickson, 2007; Galaskiewicz y Sinclair Colman, 2006; Googins et al., 2007; Korula y Laasonen, 2010; LeBer y Branzei, 2010b; Seitanidi, 2010; Wymer y Samu, 2003), pero no existe consenso para establecer tipos exclusivos u homogéneos en los cuales este continuo se manifiesta. Austin (2000; Austin et al., 2006) ha descrito tres etapas de colaboración, las que van desde la filantropía a la integración.

Otras investigaciones han puesto el acento en describir la generación de valor social y económico resultante de estas alianzas (Austin et al., 2006), de manera que puede considerarse un intangible en la medición del desempeño financiero de la compañía, siendo su contribución no negativa, es decir, al menos no es dañina y existen razones para pensar que es positiva en términos financieros (Tsoustsoura, 2004; Ruf et al., 2001). Arya y Salk (2006) ponen atención en la importancia de generar metas formales y aprendizaje en la colaboración empresas-OSFL. Especial 
importancia es puesta en el compromiso por alcanzar objetivos comunes y la generación de valor social, el cual incrementa significativamente la viabilidad y asimilación del comportamiento socialmente responsable (Arya y Salk, 2006).

\section{EL ESTUDIO}

Este artículo pretende estudiar las alianzas de cooperación de OSFL chilenas con organizaciones donantes, describiendo las principales características de dichas alianzas y su dinámica evolutiva. Para ello se ha optado por una metodología cualitativa, que permita profundizar en el cómo y por qué de las alianzas de colaboración entre empresas y OSFL. En concreto, el diseño combina el estudio de casos con la grounded theory. La elección de la muestra y definición de variables se ciñen a los principios del estudio de casos en tanto su análisis sigue los principios de la grounded theory. Estas dos metodologías son adecuadas cuando se pretende construir o contribuir a la construcción de una teoría en un área de interés poco analizada (Harrison y Freeman, 1999), como es el caso de alianzas de colaboración entre organizaciones tan distintas como las aquí expuestas. Ambas son adecuadas cuando el contexto de investigación es demasiado complejo, por el número de variables intervinientes, y resultan especialmente convenientes para el estudio de estructuras, procesos y resultados de un fenómeno (Strauss y Corbin, 2002), ya que permiten profundizar en las variables determinantes de los fenómenos analizados.

Normalmente en grounded theory no se parte de una lista predefinida de variables, sino que se deja que éstas emerjan en el transcurso de la investigación. Dado que resultaba fundamental homogeneizar las variables objeto de estudio y dado que se parte de un marco teórico previo, en vez de hacerlo así se optó por seguir las sugerencias de Eisenhardt (1999) para el diseño de casos. Esta autora sugiere que, apoyándose en la literatura previa, los investigadores deben delimitar una serie de variables relevantes, pero sin delinear de antemano la relación entre éstas. Siguiendo a Eisenhardt (1999), se formuló una lista de variables, a partir de la cual se construyó el guión de las entrevistas. Considerando el trabajo previo de Koljatic y Silva (2002), las dimensiones a explorar en estas entrevistas semiestructuradas fueron: a) orígenes de la relación (por qué surgió); b) características de la relación (recursos aportados, participación de los socios, riesgos asumidos, etc.); c) evolución en el tiempo (acciones desarrolladas, conflictos, tiempo dedicado a mantener la 
relación, etc.) y d) factores claves (dificultades, impacto percibido, aprendizajes, grado de contacto/comunicación, etc.).

La investigación fue desarrollada durante los años 2010-2011 y consideró entrevistar a gerentes o responsables de OSFL orientadas a Servicios Humanos que mantuvieran relaciones de colaboración con empresas lucrativas que ellos consideraban significativas, que tuvieran más de tres años de continuidad de operaciones, consideraron OSFL de alcance nacional como otras que poseen actividades o representaciones en otros países, pero que tienen sede en la Región Metropolitana. A partir de la clasificación de Organizaciones No Gubernamentales de Chile de la Guía Silber (Directorio de Instituciones de Chile, 2010) y teniendo presente los criterios descritos, se conformó la muestra para el estudio $(\mathrm{n}=35)$, entrevistando a un representante de cada OSFL. Para ello, se siguieron los principios del muestro teórico, cuyo objetivo es tipificar comportamientos, buscando la representatividad conceptual, no estadística (Glasser, 1978; Strauss y Corbin, 2002). Los casos no fueron elegidos por su representatividad en cuanto a tamaño, sectores, etc., sino por su aporte a la caracterización del objeto de estudio (Locke, 2001), motivo por el cual el alcance de la investigación se restringe a los casos escogidos. Con todo, la selección se realizó equilibrando el principio de igualdad con el de diferencia (Flores, 2009; Locke, 2001; Rubin y Rubin, 1995). Dentro de este grupo, el principio de diferencia se consigue porque las organizaciones elegidas son de distinto tamaño (estructura), diferenciación interna (personal profesional y voluntario) y se dedican a diversos objetivos (reparación de derechos, promoción, etc.). 
Tabla 1. Caracterización de la muestra de la investigación

\begin{tabular}{|c|c|c|c|c|c|c|}
\hline Sexo & Cargo & Presencia & $\begin{array}{c}\text { Tipo de } \\
\text { Organización }\end{array}$ & Antigüedad & $\begin{array}{l}\text { Cantidad de } \\
\text { relaciones de } \\
\text { colaboración }\end{array}$ & $\begin{array}{l}\text { Antigüedad de } \\
\text { la Relación de } \\
\text { Colaboración }\end{array}$ \\
\hline $\begin{array}{l}\text { H: } \\
\quad 56,9 \%\end{array}$ & $\begin{array}{l}\text { Gerente: } \\
59,7 \%\end{array}$ & $\begin{array}{l}\text { RM: } \\
90,3 \%\end{array}$ & $\begin{array}{l}\text { Corporación } \\
60 \%\end{array}$ & $<5$ ańos: $27,8 \%$ & $1: 27,7 \%$ & $<1$ año: $13,9 \%$ \\
\hline $\begin{array}{l}\text { M: } \\
43,1 \%\end{array}$ & $\begin{array}{l}\text { Profesional: } \\
27,8 \%\end{array}$ & Otra: $9,7 \%$ & $\begin{array}{l}\text { Fundación: } \\
35 \%\end{array}$ & $\begin{array}{l}6 \text { a } 10 \text { años: } \\
27,8 \%\end{array}$ & 2 a $4: 25 \%$ & $\begin{array}{l}2 \text { a } 10 \text { ańos: } \\
72,2 \%\end{array}$ \\
\hline & & & & $>11$ ańos: $44,4 \%$ & $<5: 47,2 \%$ & $>10$ años: $13,9 \%$ \\
\hline
\end{tabular}

Las entrevistas fueron efectuadas entre el segundo semestre de 2010 y el primer semestre de 2011. Se grabaron guardando recaudo del consentimiento informado, transcritas íntegramente y codificadas utilizando el software atlas.ti 5.2, siguiendo las directrices de la grounded theory (Valles, 2000; Flores, 2009). Para determinar la fiabilidad de los resultados, Strauss y Corbin (2002: 253) proponen varios criterios. Uno de ellos es la consistencia entre codificadores. En este sentido, se contrataron codificadores independientes que analizaron separadamente sus entrevistas, siendo la coincidencia inicial de categorías del 95\%. Las divergencias se resolvieron analizando de nuevo las entrevistas y justificando con base en las declaraciones de los participantes, los matices que cada codificador había establecido. Para la selección de los códigos y sus respectivas citas en la presentación de los resultados, se consideraron aquellos que obtuvieron mayor mención (grounded) en las unidades hermenéuticas analizadas en cada entrevista ( $>5$ por entrevista) y entreentrevistas (>100 en la unidad hermenéutica). La selección final de códigos y citas -que se presenta en este artículo- se hizo bajo criterio experto, buscando recaudar de mejor manera el sentido expresado por los entrevistados y su vinculación con la teoría. Estos códigos (o conceptos) fueron agrupados en categorías (codificación axial), apoyándonos en la literatura como primera unidad de análisis (Strauss y Corbin, 2002), por lo cual la presentación de los temas aquí expuestos obedecen a la combinación parsimoniosa de lo expresado por la literatura, la emergencia de los temas indicados por los entrevistados y la densidad de la codificación antes mencionada, tal como lo proponen la metodología escogida (Glasser, 1978; Valles, 2000; Locke, 2001; Strauss y Corbin, 2002; Flores, 2009). 


\section{PRINCIPALES RESULTADOS}

Los resultados obtenidos se agrupan en un esquema conceptual construido de acuerdo a las dimensiones sugeridas por Koljatic y Silva (2002), corregidas y actualizadas con los mismos resultados Eisenhardt (1999):

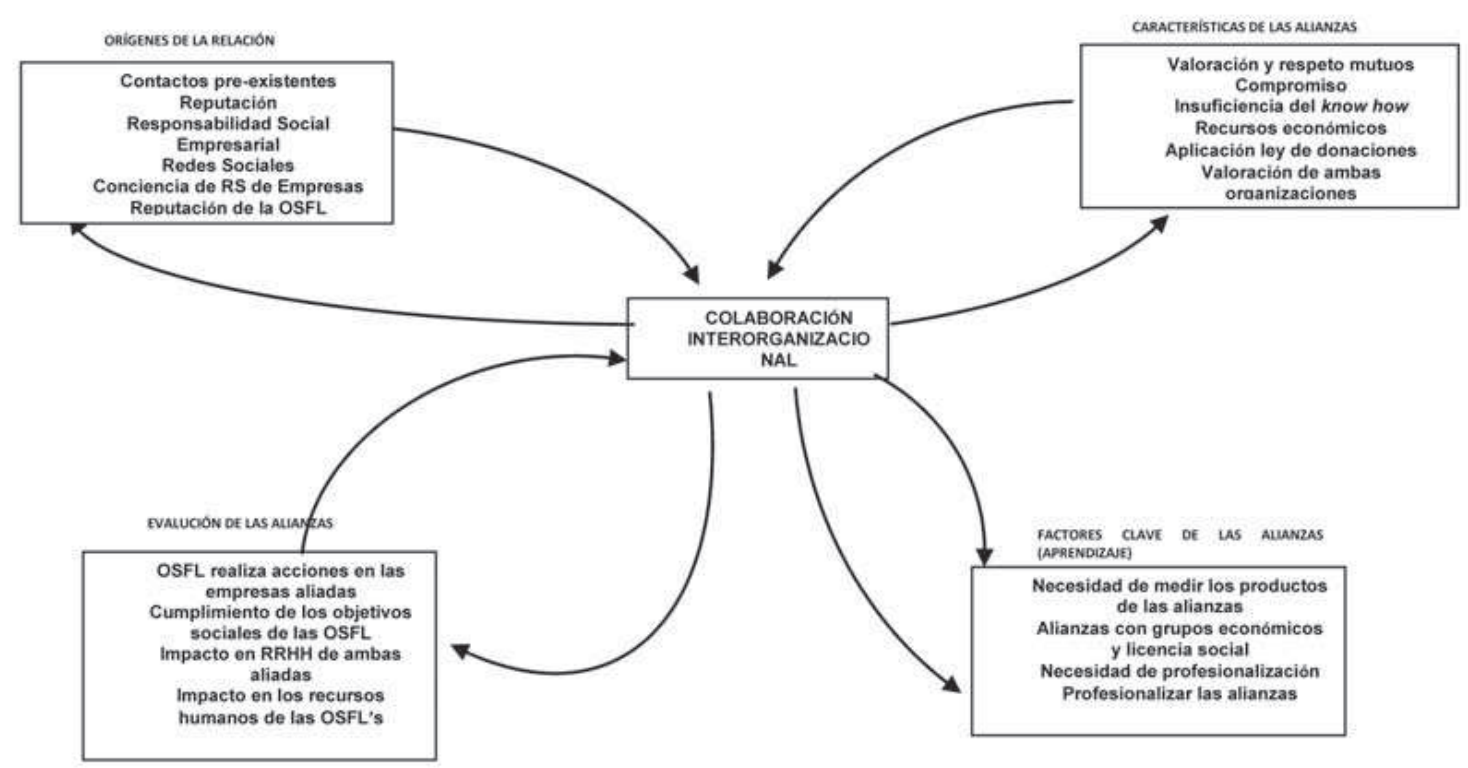

\subsection{Origenes de la relación}

La mayor parte de los entrevistados indica que las alianzas surgen a partir de contactos pre-existentes que hacen posible instaurar el vínculo. Como señalaran Koljatic y Silva (2002), este tipo de relaciones incluye vínculos sociales primarios entre gerentes o encargados de las OSFL y gerentes o encargados de llevar adelante la Responsabilidad Social de las empresas. Ellos pueden tener lazos de parentesco, haber sido compañeros de colegio o Universidad, o haber frecuentado los mismos círculos sociales, vale decir constituyen un tipo particular de redes sociales. La importancia de esta clase de redes parece tener raíces culturales (Rodríguez y Quezada, 2007).

"Cuando nos toca abordar ciertas empresas y se exponen en el fondo "éstas son las empresas que queremos atacar", y tú dices, "de acuerdo, yo tengo un conocido, yo tengo una persona de afinidad, yo conozco a fulanito de tal y asi un poco se va distribuyendo, cosa de que la empresa sea abordada por la persona que tiene mayor probabilidad de éxito". 
"La relación con (la empresa) nace, cuando (la Fundación) estaba empezando, a raíz de contactos directos del directorio".

"Entonces a ese nivel hay una doble búsqueda, a veces ellos nos han venido a buscar o nosotros mismos tratamos de persuadirlos, entrando a través de alguien, ahora esos convenios son siempre, se tienen que negociar a alto nivel, no son convenios en los cuales uno pueda entrar llamando por la guía de teléfonos, ubicándolos".

Además de este rasgo cultural, los entrevistados indican que la generación de alianzas se facilita por la reputación o reconocimiento social. En este sentido, OSFL que poseen una alta valoración social son preferidas por las empresas privadas para establecer convenios.

"El capital social se distribuye de manera desigual en muchos otros capitales y esa es una realidad indiscutible, instituciones que evidentemente tiene un enorme capital social en comparación con otras instituciones que tienen mucho menos, van a tener acceso a poder gestionar recursos, generar empresas y vinculos significativos".

Por esta razón, las OSFL destacan sus logros como un factor que impregna sus alianzas de colaboración.

"(la Fundación) ya está instalada, es una maravilla, yo voy a otro lugar y todo el mundo nos conoce, una fundación buena, responsable, ordenada, ejecutiva, con todo lo que implica".

La reputación de las organizaciones sin fines de lucro es considerada un factor que no sólo ayuda a la conformación de alianzas con las empresas, sino que también es un resultado de las mismas, de ahí su vital importancia. Por este motivo, las OSFL estudian cuidadosamente a sus eventuales patrocinadores y la imagen que proyectan sobre sus voluntarios, sus beneficiarios y otros donantes efectivos o potenciales.

\footnotetext{
"Por supuesto nosotros nos tenemos que mantener, porque (la Fundación) tiene varios clientes no nos interesa solamente tener buena relación con las empresas, o sea las relaciones con las empresas no pueden ser a costa de lo atractivo que puede ser el Techo para que ingresen nuevos voluntarios, no puede ser a costa de la imagen que tienen los pobladores de los campamentos respecto de esta Institución, cuya prioridad va a ser siempre jugársela por el futuro de ellos y que esto no va a estar distorsionado por una empresa...".
}

Otro elemento facilitador del surgimiento de las alianzas está relacionado con el interés de ciertas empresas por conformarlas. La aparición relativamente 
reciente de este interés se deriva de condiciones de la moderna sociedad mundial (Luhmann, 2007). Algunas OSFL indican que en las empresas se está produciendo un clima favorable a la colaboración con ellas, debido a una mayor conciencia de responsabilidad social empresarial.

"En los últimos 15 o 20 años, yo creo que ha habido una evolución a que las empresas están mucho más interesadas, ya no solamente me interesa colaborar contigo en términos económicos, sino que me interesa colaborar contigo mucho más alla".

\subsection{Caracteristicas de las alianzas}

Independientemente de su origen, las alianzas modernas de colaboración se caracterizan por su mayor pragmatismo. En este sentido, una exitosa gestión realizada por las OSFL en proyectos y programas sociales permite un reconocimiento social extendido. Ello implica que la organización del tercer sector es capaz de demostrar, ante las empresas donantes, una adecuada gestión de los recursos económicos obtenidos y el cumplimiento de los objetivos planteados en las acciones sociales financiadas por las empresas. Esto también tiene un efecto positivo sobre empresas privadas que buscan alianzas que les faciliten lograr los requerimientos sociales de Responsabilidad Social Empresarial.

"Yo creo que parte de nuestro mayor valor es que es una fundación que genera confianza y ahi, yo creo que eso no está en duda".

"verdaderamente se hace lo que se dice que se va a hacer, sobre todo en el manejo de las platas, de que efectivamente los recursos que se están entregando van de manera eficiente y eficaz a golpear concretamente la vida de determinados segmentos de la población con los cuales nosotros estamos trabajando".

Las relaciones de colaboración que tienden a permanecer en el tiempo son aquellas en las cuales se produce un involucramiento activo de ambas organizaciones en torno a los proyectos conjuntos.

"Tenemos el programa de tutorias, donde tenemos gerentes de primera linea de (la empresa) que se juntan una vez al mes con los niños de cuarto medio nuestro".

"Tú vas ayudando al sector empresarial a focalizarse y desarrollar programas educativos, por el tema de responsabilidad social más consistente". 
Otra característica de las alianzas de colaboración perdurables es que pasa a ser valorada como un factor de éxito. Esto quiere decir que un elemento mediador del éxito de la colaboración entre el mundo social y el empresarial consiste en la valoración y respeto mutuos, lo que permite ir afianzando la confianza para el desarrollo de la colaboración.

"Mira, la sociedad privada puede ser parte de la solución de la pobreza, puede ser parte de la integración de la sociedad, para nosotros eso es súper importante".

Desde el punto de vista legal, la ley de donaciones promueve que las empresas privadas inviertan en proyectos sociales, deduciendo parte de la inversión de sus impuestos. Este es uno de los elementos que permite a las organizaciones no lucrativas acercarse de mejor manera a emprender programas sociales.

"Lo que pasa es que la ley de donaciones con fines educacionales tiene la gracia de que tú puedes donar y se te devuelve el 50\% del capital donado, entonces para las empresas es súper atractivo, es un gancho para las empresas".

"Lo que pasa es que al final... la cantidad de plata que tienes es la cantidad de cosas que puedes hacer".

De esta manera el estado estimula la relación entre OSFL y empresas donantes incrementando, en una forma atractiva para ambas clases de organizaciones, los recursos económicos de que dispone la organización social para realizar sus actividades. Los aportes financieros condicionan el alcance de los programas y proyectos de las OSFL y las empresas lucrativas disminuyen su carga impositiva, por lo que las alianzas de colaboración se vuelven realmente importantes. Entre las condiciones que caracterizan la situación actual de las alianzas de colaboración, también se encuentran dificultades derivadas de la falta de experiencia en el trato entre organizaciones que, como vimos, operan con racionalidades tan diferentes. Todavía no se ha desarrollado plenamente un know how de la colaboración, lo cual queda en evidencia por la ausencia de estándares o pautas que ayuden a otras OSFL a iniciar alianzas de colaboración con empresas lucrativas. Otro indicador de esto es la inexistencia de planificación y proyección a largo plazo de las alianzas. La insuficiencia de know how también se expresa en la diferencia de expectativas entre organizaciones sociales que quisieran proyectar la alianza en el tiempo, mientras las empresas desean desplegar instancias de colaboración puntuales y 
acotadas en el tiempo.

\subsection{Evaluación de las alianzas}

Como todo vínculo social, según transcurre el tiempo, las alianzas de colaboración van siendo evaluadas por sus integrantes. De aquí se desprende un aprendizaje que no se debería perder. En esta dimensión temporal, muchos de nuestros entrevistados indican que las OSFL también desarrollan actividades para las empresas donantes, como puede ser la prestación de servicios ligados a su propia experticia. Algunas fundaciones, por ejemplo, realizan charlas y programas de prevención en el consumo de drogas para los empleados que trabajan en las empresas donantes, lo cual enriquece el vínculo de colaboración.

\footnotetext{
"Este es el programa que va, se instala en la empresa, se invita a trabajadores que están en un estado bien de burnout, y que ya de repente, se los invita a una charla motivacional; a un grupo grande de gente, de ahi surge gente que está motivada, que puede reflexionar y, en una de esas, está dispuesta a dejar el tabaco".
}

Las organizaciones sin fines de lucro entienden las relaciones de colaboración con las empresas como una vía que permite el desarrollo o mejoramiento de la sociedad en general, abordando problemáticas en conjunto. Es posible señalar que, por medio de las alianzas observadas, las OSFL persiguen dar cumplimiento a objetivos sociales referidos al desarrollo y cohesión social en diversas de áreas. Así, distintas OSFL destacan que las alianzas de colaboración permiten mejorar habilidades de inserción laboral en discapacitados o generan un impacto real en la vida de personas económicamente necesitadas.

Dado que en esta investigación se entrevistó a gerentes y representantes de organizaciones sociales, se recogió abundante información acerca del impacto que tiene la instauración de alianzas para los recursos humanos de las mismas OSFL y también para los empleados de las empresas lucrativas. Ese impacto positivo se expresa en:

I. Captación de nuevos socios. Los representantes de OSFL entrevistados indican que la alianza de colaboración ha contribuido a que la fundación capte nuevos socios. 
"(la empresa) siempre nos ha apoyado en todo lo que son nuestras campañas de socios, que hacemos una vez al año, levantamos 50.000 socios, buscamos levantar 50.000 socios al año y ellos nos colaboran en toda la plataforma tecnológica".

II. Aumento en la motivación de los trabajadores. Si bien no se cuenta con indicadores directos, la estabilidad de las alianzas de colaboración parece producir un aumento en la motivación de los trabajadores de ambas organizaciones aliadas.

"Se produce mayor motivación cuando se materializan nuevas alianzas".

III. Traspaso de recursos humanos calificados. En algunas oportunidades, la relación de colaboración entre ambas organizaciones permite el intercambio de recursos humanos como expresión de profundización de la alianza.

IV. Mejoramiento del clima laboral de las empresas lucrativas. Algunas organizaciones sociales sostienen que las alianzas de colaboración tienen una incidencia positiva y directa en el clima laboral de las empresas donantes, generando, además, compromiso de empleados de la empresa donante con los principios de la OSFL.

"Como ha sido esta relación particularmente con el Banco, profundamente, también la tuvimos con la Financiera ponte tú, después con el Banco, pero vinculos trascendentes, trascendentes, y de gente que después ha dejado la empresa y que ha seguido vinculada con nosotros por un tema de pertenencia".

V. Mayor estabilidad laboral. Las entrevistas también reconocen que las alianzas permiten una mayor estabilidad laboral de los empleados de las organizaciones sociales.

"Es que como tenemos un aporte económico, eso nos ayuda a estabilizar todas nuestras contrataciones".

\subsection{Aprendiendo factores clave de las alianzas de colaboración}

Las evaluaciones señaladas en el punto anterior se complementan con relevantes reflexiones de nuestros entrevistados que apuntan al desarrollo de factores claves para facilitar las alianzas de colaboración con las empresas, potenciando sus efectos positivos. 
Necesidad de medir la evolución y resultados de la alianza. Del mismo modo en que no se dispone de sistemas de medición de los programas y proyectos sociales ejecutados, tampoco se cuenta con instrumentos que permitan medir el desarrollo y resultados de las alianzas de colaboración. Esta es una tarea que debiese ser abordada a futuro.

"Hay que medirla desde la perspectiva de las empresas, porque ese, es en el fondo, es el beneficio o el gran beneficio que tiene apoyar a una institución de beneficencia".

"Pero también nosotros si gastamos un poco de plata en evaluar la factibilidad, tampoco les pasamos la boleta, como que desde la buena intención nos hicieron una petición, nosotros la petición la evaluamos y desde ahi nos juntamos a conversar después, yo creo que por lo mismo, el voluntario se tuvo que devolver antes, nosotros fuimos y les explicamos por lo menos, "oye mira pasó esto y esto, no es lo ideal, nos gustaría que te devolvieras antes porque no estás haciendo un buen trabajo", nos hace generar expectativas falsas también y ellos ahi también tuvieron ese mismo como colchoncito de decir "pucha la lata, mm... sí, pero bueno".

Conveniencia de establecer alianzas con grupos económicos que garanticen la continuidad de la labor de las OSFL. Muchas de las organizaciones entrevistadas indican que la consolidación de su plan de alianzas de colaboración les ha permitido más que buscar alianzas con organizaciones particulares, realizar la búsqueda con grupos económicos consolidados. Ello garantizaría continuar con la labor social desplegadas por la OSFL.

"De ahi fluye mucho más fácil ejecutar cosas y hacer cosas y tenemos una variedad de empresas "dispuestas", con una cabeza que dice: "si, yo quiero apoyar".

De gran importancia para las empresas con afán de lucro es que la colaboración con OSFL contribuye directamente a que obtengan la licencia social para operar en lugares o comunidades específicas. Considerar esta ventaja para las empresas como un logro de la colaboración puede contribuir, además, a que se amplíe la oferta de programas y proyectos ejecutados por la fundación, lo que también es un valor específico producto de este encuentro entre empresa y mundo social.

"Si, claro, eso se ha dado. Nos ha dado espacios para poder hacer mejoras, evaluar nuevas coberturas, tipos de intervención". 
Necesidad de profesionalizar las alianzas. Un modo de asegurar el éxito y perduración de las alianzas consiste en definir mayores y mejores estándares que orienten las actividades en común y aseguren el logro de los objetivos acordados generando, además, formas innovadoras que puedan hacer la colaboración cada vez más interesante.

Para profesionalizar las alianzas, se menciona una serie de acciones posibles de realizar:

I. Importancia de un comité ejecutivo que gestione las alianzas. Algunas OSFL entrevistadas indican que un factor relevante en sus relaciones de colaboración con empresas ha sido la formación de un comité que se establece junto a la empresa, para gestionar las alianzas. Ello permite una mayor integración y colaboración en conjunto.

II. Equipo paritario. También es recomendable llevar a cabo un trabajo conjunto e integrado con la empresa para sacar adelante los programas y proyectos, tal como un equipo "paritario" integrado por representantes de ambas organizaciones.

"yo estoy trabajando todo el tema con las empresas donde hay un representante nuestro y un representante de ellos, a veces uno de ellos y a veces está dividido en áreas también, en las áreas de desarrollo que tengas con cada uno de ellos".

III. Planificación de las alianzas. En algunos casos, el proceso evolutivo de las alianzas de colaboración ha llegado a un momento en que es necesario planificar, en conjunto con las empresas, las acciones sociales. Ello implica reconocer que la alianza se encuentra en otro momento, que no es de petición exclusiva de dinero, sino de desarrollo de programas en conjunto.

"Sin embargo, por ejemplo con (la empresa), acá es el detalle, pero la semana laboral, prácticas laborales, becas universitarias, charlas magistrales, charlas técnicas, tutorias, tenemos nuestro programa todo desarrollado".

IV. Fidelización de empresas aliadas. Especial importancia tiene que las organizaciones del sector social logren consolidar relaciones estrechas y permanentes con las empresas donantes, de manera tal que los programas y proyectos que desarrollan en conjunto permitan fidelizar la acción social de la empresa. 
"De la fundación surgen necesidades, de fidelizarlos a ellos, como que es su proyecto, pese a que muchas de esas respuestas las tenemos nosotros".

V. Nuevas acciones producto de la alianza. Un elemento destacable de las relaciones de colaboración es que han permitido ir generando nuevos programas y proyectos a partir de la colaboración inicial. De este modo va cambiando la dinámica de la alianza, introduciendo elementos dinamizadores en la relación.

"Yo he visto un cambio cualitativo, te diría yo, de la relación de (la Fundación) con el mundo empresarial o las organizaciones empresariales. Básicamente te diría que la modificación se traduce en una mucho mayor preocupación, mucho mayor involucramiento, una mucho mayor participación de tanto la empresa como sus trabajadores, respecto del quehacer concreto de las instituciones".

VI. Institucionalización de la comunicación. Para monitorear efectivamente las acciones desarrolladas, algunos entrevistados señalan que una comunicación institucionalizada por medio de varios canales e instancias permite desplegar de mejor manera el vínculo instaurado con la empresa.

"El inicio del año, el final del año con un informe de presentación y un informe final, en forma permanente una vez al mes y mucha cosa por e-mail y teléfono, hay un sistema de gestión bastante permanente".

VII. Profesionalizar la petición. Es preciso que las organizaciones del mundo social aprendan a formular su petición de recursos de un modo que encuentre sentido en las empresas, lo que pasa necesariamente por establecer un lenguaje en común. Las empresas son organizaciones con fines de lucro cuyo objetivo básico es la obtención de recursos para la reproducción del sistema. Como hemos visto al caracterizar los tres sectores en que se desempeñan los distintos tipos de organizaciones, las racionalidades que orientan el quehacer de las organizaciones se derivan del sector al que pertenecen. El aprendizaje que deben hacer las OSFL consiste en mostrar que la alianza con una empresa lucrativa puede agregar valor económico a dicha empresa, del mismo modo como debe agregar valor social que sea atractivo para la OSFL. La licencia social, para sólo señalar un ejemplo evidente, agrega un valor económico tan importante, que sin ella ninguna empresa puede operar. 
"Porque también las fundaciones sociales tenemos que estar mirando al mundo y el sector empresarial te abre un lenguaje que es importante".

\section{CONCLUSIONES}

Las organizaciones se observan a sí mismas y a su ambiente haciendo uso de distinciones que constituyen lo observado. En esto consiste la construcción de sentido que las organizaciones hacen de sí mismas y del entorno (Luhmann, 2011). En el caso que nos interesa, las organizaciones privadas utilizan la distinción lucro/no lucro, considerando el lucro como el lado relevante, que otorga sentido, y dejando inobservado el lado del no lucro, como trasfondo irrelevante. Las organizaciones sin fines de lucro, por su parte, también usan esa distinción, pero para ellas el lado relevante es no lucro, en tanto el lucro queda como trasfondo irrelevante. Es evidente, entonces, que el entorno construido por ambos tipos de organización no es el mismo y que, por decirlo así, viven en mundos distintos. Que el lucro sea relevante significa que, para las organizaciones lucrativas, es indiferente todo lo que no pueda ser visto en esos términos y, por esa razón, es preciso utilizar ese código para llegar a establecer una comunicación con ellas. Dado que, por su parte, las OSFL se definen negativamente, el lucro no puede ser su código. Se hace comprensible que el diálogo entre ambas clases de organizaciones sea difícil y que su comunicación sea improbable. Sin embargo, no es imposible. A diferencia de las definiciones afirmativas, que sólo admiten lo que su predicado explícitamente permite, las definiciones negativas únicamente excluyen lo que su negación indica, dejando abierto un ámbito mucho más grande de posibilidades. Que una organización sea "sin fines de lucro", por ejemplo, sólo impide considerar al lucro como indicador relevante para observarse a sí mismas en relación con su entorno, pero no excluye la eficiencia ni la optimización de recursos tras el logro de sus fines. El diálogo encuentra, de este modo, una vía posible entre ambos tipos de organización. De acuerdo a los resultados obtenidos en esta investigación, las OSFL deberán procurar que la organización lucrativa vea oportunidades de lucro en su relación con la OSFL, sea ello entendido como rebajas impositivas, mejoría de imagen, marketing, licencia social, apoyo especializado para los trabajadores, etc. Muchas de estas acciones han sido documentadas por la literatura sobre esta materia como ventajas de las alianzas de colaboración (Inkpen, 2002; Jamali y Keshishian, 2009). 
Con todo, las OSFL deberán indicar con claridad los costos económicos que la asociación tendrá para la organización lucrativa y, finalmente, deberá estar en condiciones de garantizar que los fondos otorgados no serán malgastados en una operación burocrática excesiva o en desvío de fondos para fines que no habían sido explícitamente anunciados. Nada de esto implica una pérdida de autonomía para la OSFL, ni tampoco la distorsión de su misión, la cual sigue sumida en la recursividad de su autopoiesis (Luhmann, 2011). Ella continuará orientando su quehacer por fines no lucrativos, aunque probablemente aprenderá que la eficiencia en el uso de sus recursos, la profesionalización de la gestión y el uso de mecanismos de control de gestión pueden contribuir enormemente al logro de su finalidad no lucrativa. Ello comienza a ser asumido lentamente. Nuestros hallazgos nos indican que la profesionalización de la gestión no lucrativa no encuentra aún necesario el establecimiento de mediciones más exhaustivas de resultado ni de impacto de sus intervenciones. La distinción no lucro parece encontrarse anclada, en las organizaciones consideradas en esta investigación, en tradiciones filantrópicas inspiradas en la caridad cristiana. La idea de una causa justa, de reparación de derechos o su promoción, parece ser suficiente como para no desarrollar estrategias de accountability.

Nuestros resultados demuestran que las alianzas ayudan a que ambas organizaciones aliadas puedan seguir desarrollando su autopoiesis y no pierdan el referente que el mandato de su misión les ha impuesto. Cada una de ellas parece encontrar ventajas en estas alianzas, que reintroduce en su operatoria entendida como un mejoramiento del clima laboral, el hecho que las OFSL se hagan más atractivas para sus socios, estén en condiciones de ofrecer mayor estabilidad en el empleo y descubran la importancia de profesionalizar su gestión y la forma de relacionarse con las empresas donantes.

Por otra parte, actualmente, en nuestro país, las OSFL asumen la provisión de nuevos servicios con alta eficiencia en su capacidad de gestión, pues su función en el sistema social no solamente es ser portadoras de un espíritu solidario o canales de altruismo, en sí una función destacable, sino además, proveedoras de servicios a públicos crecientemente segmentados en sus necesidades y demandas, que tienen una rápida dinámica de cambio y que deben legitimarse ante el Estado y el sector empresarial como organizaciones válidas, sobre todo en su capacidad de gestión, 
altamente efectiva, modernizada y profesional (Rodríguez, 2001).

Las organizaciones lucrativas también han cambiado su perspectiva en la distinción lucro/no lucro. La indiferencia con que consideraban el lado del no lucro las hacía estimar de escaso interés relacionarse con organizaciones no lucrativas. Sin embargo, la sociedad se ha tornado mundial y está experimentando una profunda transformación (Luhmann, 2007), algunos de cuyos indicadores, como los cambios en las sensibilidades frente al medio ambiente, la creciente conectividad desarrollada por los individuos gracias a las nuevas tecnologías de la información, el aumento del poder de los clientes, el incremento de la capacidad de observación por parte de la sociedad mundial y la correspondiente exigencia de transparencia han llevado a que las organizaciones con fines de lucro amplíen sus audiencias significativas, cambiando de la restringida preocupación por responder a sus shareholders a la amplia necesidad de atender múltiples stakeholders. Como consecuencia de esto, el lado del lucro se ha debido redefinir, para descubrir oportunidades de lucro en ámbitos antes desechados como no lucrativos o bien, lo que no es menos importante, para tomar conciencia que el descuido del valor social puede repercutir gravemente en la pérdida de valor económico. En los términos que nos interesan, esto ha significado que la relación con organizaciones no lucrativas, que antes parecía irrelevante y se evitaba completamente o se restringía a los marcos de la filantropía, aparece bajo un prisma lucrativo que la hace ver interesante, por ejemplo, integrando una estrategia de marketing (Froelich, 1999: 252). Por otra parte, las influyentes ideas de Peter Drucker acerca de convertir la gestión en una profesión capaz de hacerse responsable por el bienestar y no sólo por la riqueza, agregando que la gestión era una vocación honorable si asumía responsabilidades más amplias, entre las que podía estar la colaboración con empresas no lucrativas, vuelven a ser recordadas por los especialistas (Kanter, 2009).

La colaboración entre empresas con afán de lucro y OSFL puede observarse, entonces, bajo un nuevo prisma. Las organizaciones con fines de lucro mejoran su imagen ante sus stakeholders, entre los cuales se debe destacar la comunidad en que están insertas, la opinión pública general, los clientes y también sus propios empleados, quienes muestran mayor disposición a comprometerse con una organización que apoya actividades altamente valoradas por la sociedad; este mejoramiento de la imagen contribuye, además, a atraer postulantes de mejor 
calidad y a enriquecer la cultura organizacional. Las OSFL no sólo consiguen mayor estabilidad para el flujo de sus recursos financieros, sino que adquieren los conocimientos de administración necesarios para hacer una mejor gestión de aquellos; la alianza les otorga, adicionalmente, el reconocimiento por parte del mundo económico que les abre nuevas oportunidades de acceso a fuentes de financiamiento y, con ello, mayor cobertura en el cumplimiento de su misión (Austin, 2000). Las OSFL encuentran además, en la alianza, nuevas posibilidades de generación de valor, mostrando que la acción social puede contener una coherencia financiera, una nueva mirada sobre compartir riesgos y responsabilidades respecto del entorno social, una visión pragmática sobre los fenómenos sociales y su vinculación e impacto con el desempeño empresarial.

Nuestro estudio constató que las relaciones de colaboración desarrolladas en estos casos, se basan fuertemente en factores culturalmente afianzados, principalmente en nexos interpersonales ya establecidos, centrados en la confianza (amistad o relación de parentesco), tal como ha sido indicado por la literatura internacional, siendo un factor reductor de incertidumbre (Arino et al., 2005; Pitsis et al., 2005; Wohlstetter et al., 2005). No obstante, el Estado ha comenzado a incentivar la formación de este tipo de alianzas a través de leyes como la de donaciones y los cambios de sensibilidad en la sociedad mundial hacen necesaria la obtención de licencia social, lo que incrementa el interés de las empresas lucrativas por aliarse con OSFL. Los hallazgos encontrados a partir de los casos que hemos considerado en esta investigación nos indican que las relaciones de colaboración efectivas producían un compromiso con la misión y causa social de la OSFL. No obstante, la relación de colaboración no se proyectaba más allá del corto plazo y dependía mucho de la atención y energía que brindaban los líderes de ambas organizaciones involucradas (OSFL y empresas). Tal como lo indica Austin (2000) las alianzas son vehículos para cumplir con la misión de cada uno de sus participantes, lo cual significa que deben ser consideradas como parte integral de la estrategia tanto de las OSFL como de las organizaciones donantes.

Hemos elaborado, en síntesis, un modelo que incorpora las variables que han de ser evaluadas para estudiar esta clase de alianzas considerando su dinámica, no en los términos lineales propuestos por Austin y que no fueron corroborados por nuestro estudio, así como tampoco por el de Koljatic y Silva (2002), sino 
en términos que permitan comprender que todas las variables y cada una de ellas pueden experimentar variaciones derivadas de la misma alianza y también las que se originen en el entorno social. A partir de los casos estudiados en esta investigación, podemos afirmar que las tres etapas del continuo propuesto por Austin parecen ser más bien una tipología ideal y no una vía probable de evolución de esta clase de alianzas.

\section{REFERENCIAS}

Arino, Africa; De la Torre, José y Ring, Peter. "Relational Quality and Interpersonal Trust in Strategic Alliances", European Management Review 2 (2005):15-27.

Arya, Bindu y Salk, Jane. "Cross Sector Alliances Learning and Effectiveness of Voluntary codes of Corporate Social Responsibility", Business Ethics Quarterly 16/2 (2006): 211-234.

Austin, James y Seitanidi, María. "Collaborative value creation: A review of partnering between nonprofits and businesses: Part I. Value creation spectrum and collaborations Stages". Nonprofit and voluntary Sector Quarterly. Publisehed online 16 July 2012. DOI 10.1177/089976401250777(2012).

Austin, James. "Strategic Collaboration between Nonprofits and Business", Nonprofit and Voluntary Sector Quarterly 29/1 (2000): 69-97.

Austin, James. El desafío de la colaboración. Buenos Aires: Granica, 2003.

Austin, James; Gutiérrez, Roberto; Ogliastri, Enrique y Reficco, Ezequiel. Gestión efectiva de emprendimientos sociales. Lecciones extraidas de empresas y organizaciones de la sociedad civil iberoamericana. México: Planeta, 2006.

Bouwen, René y Taillieu, Tharsi. "Multi-party Collaboration as Social Learning for Interdependence: Developing Relational Knowing for Sustainable Natural Resource Management", Journal of Community \& Applied Social Psychology 14 (2004):137-153. 
Brickson, Shelley. "Organizational identity orientation: The genesis of the role of the firm and distinct forms of social value", Academy of Management Review 32 (2007): 864-888.

Brinkerhoff, Jennifer. "Government-Nonprofit Partnership: A Defining Framework", Public Administration and Development 22 (2002): 19-30.

Charmaz, Kathy. Constructing Grounded Theory: A Practical Guide Through Qualitative Analysis. CA: Sage, 2006.

Drucker, Peter. Managing the Non-Profit Organization. New York: Harper Business, 1992.

Eisenhardt, Kathleen. "Building Theories from Case Study Research", Academy of Management Review 14/4 (1999): 532-550.

Flores, Rodrigo y Donoso, María. "Organizaciones del Tercer Sector: avanzando en la necesidad de caracterizar el voluntariado chileno”, Revista de Trabajo Social 81(2012): 69-78.

Flores, Rodrigo. Observando observadores: una introducción a las técnicas cualitativas de investigación social. Santiago: Ediciones Universidad Católica, 2009.

Foster, Mary y Meinhard, Agnes. "A Regression Model Explaining Predisposition to Collaborate", Nonprofit and Voluntary Sector Quarterly 31/4 (2001): 549564.

Froelich, Karen. "Diversification of Revenue Strategies: Evolving Resource Dependence in Nonprofit Organizations", Nonprofit and Voluntary Sector Quarterly 28/3 (1999): 246-268.

Galaskiewicz, Joseph y Sinclair Colman, Michelle. "Collaboration between corporations and nonprofit organization”. En Richards Steinberg y Walter Powel (eds.). The non-profit sector: A research handbook. New Haven, CT: Yale University Press (2006): 180-206. 
Glasser, Barney y Strauss, Anselm. The Discovery of Grounded Theory: Strategies for Qualitative Research. Chicago: Aldine Publishing, 1964.

Glasser, Barney. Theoretical Sensitivity: Advances in the Methodology of Grounded Theory. Mill Valley, CA.: University of California, 1978.

Googins, Bradley; Mirivis, Philip y Rochlin, Steven. Beyond good company: Next generation corporate citizenship. New York, NY: Pagrave MacMillan, 2007.

Harrison, Jeffrey y Freeman, R. Edward. "Stakeholders, Social Responsibility, and Performance: Empirical Evidence and Theoretical Perspectives", Academy of Management Journal 42 /5 (1999): 479-485.

Inkpen, Andrew. "Strategic Alliances". En Michael Hitt, R. Edward Freeman y Jeffrey S. Harrison (eds.). The Blackwell Handbook of Strategic Management. Blackwell Publishing, Cornwall (2002): 409-432.

Jamali, Dima y Keshishian, Tamar. "Uneasy Alliances: lessons Learned from Partnerships Between Businesses and NGOs in the context of CSR", Journal of Business Ethics 84 (2009): 277-295.

Kanter, Rosabeth. "Lo que nos diría Peter", Harvard Business Review (2009): 57-63.

Koljatic, Mladen y Silva, Mónica. "Uncertainty reduction mechanisms in cross-sector alliances in Latin America”, Journal of Business Research 61(2008):648-650.

Koljatic, Mladen y Silva, Mónica. "Variables asociadas a experiencias de colaboración entre empresas con y sin fines de lucro: Un estudio de casos”, Revista Abante 1/5 (2002).

Kolk, Ans; Van Tulder, Robert; Kostwinder, Esther. "Partnership for Development", European Management Journal 26/4 (2008):262-273. 
Kourula, Arno y Laasonen, Salla. "Nongovernmental organizations in business and society, management, an international business-Review and implications 1998-2007”, Business \& Society 49/1(2010a):3-5.

LeBer, Marlene y Branzei, Oana. “(Re)forming strategic cross-sector partnerships: relational processes of social innovation”, Business \& Society 49/1 (2010b):140-172.

Locke, Karen. Grounded Theory in Management Research. CA.: Sage, 2001.

Luhmann, Niklas. La sociedad de la sociedad. México: Herder, 2007.

Luhmann, Niklas. Organización y decisión. México: Herder, 2011.

Mangili, Jobbani. "Evolución del Sector Sin Fines de Lucro en el Mundo: Oportunidades y Desafíos para Chile". Tesis de Grado MBA. Valparaíso: Universidad Técnica Federico Santa María, 2005.

Pitsis, Tyrone; Josserand, Emmanuel; Clegg, Stewart; Kornberger, Martín. "Making interorganizational relationship work: an introduction", Management 8/4(2005): 69-72.

PNUD-FOCUS. Estudio Comparativo del Sector sin Fines de Lucro. Santiago de Chile: John Hopkins Univeristy. Programa de Naciones Unidas para el Desarrollo, 2006.

Porter, Michael y Kramer, Mark. "La creación de valor compartido", Harvard Business Review (2011): 32-49.

Radrigán, Mario; Dávila, Ana María; Penaglia, Francesco. "Gestión y Liderazgos en los emprendimientos sociales: el caso del sector no lucrativo chileno”, Polis [En línea] 32 (2012). Disponible en: <http://polis.revues. org/6535;DOI :10.4000/polis.6535>. [consultado: el 24 octubre 2012]. 
Rodríguez, Darío y Quezada, Soledad. "Cultura en organizaciones del tercer sector chileno", Revista Española del Tercer Sector 6 (2007).

Rodríguez, Darío y Ríos, René. "Las organizaciones sociales en una sociedad compleja”. En Luis Moya (ed.). Gobernar los Cambios: Chile, más allá de la crisis. Santiago de Chile: LOM Ediciones (2002): 133-152.

Rodríguez, Darío. Gestión organizacional: Elementos para su estudio. $1^{\circ}$ edición. Santiago de Chile: Pontificia Universidad Católica de Chile, 2001.

Rubin, Herbert y Rubin, Irene. Qualitative Interviewing: The Art of Hearing Data. Thousand Oaks, CA.: Sage, 1995.

Ruf, Bernadette; Multalidhar, Krishnamurt; Brown, Robert; Janney, Jay y Paul, Karen. "An empirical investigation of the realtionship between Change in Corporate Social Performance and Financial Performance: A Stakeholder Theory Perspective", Journal of Business Ethics 32/2(2001): 143-156.

Samii, Ramina; Van Wassenhove, Luk ; Bhattacharya, Shantanu. "An Innovative Public Private Partnership: New Approach to Development", World Development 30/6 (2002): 991-1008.

Seitanidi, María. Annual Review of Social Partnerships Issue 6( 2011). Disponible en: < http://works.bepress.com/may_seitanidi/32>.

Seitanidi, María . The Politics of Partnerships. Berlin: Springer, 2010.

Strauss, Anselm y Corbin, Juliet. Bases para la investigación cualitativa. Medellín: Editorial Universidad de Antioquia, 2002.

Tsoutsoura, Margarita. "Corporate Social Responsibility and Financial Performance. Center for Responsible Business", Working Paper Series 7 (2004). Disponible en: http://www.haas.berkeley.edu/responsiblebusiness/ documents/2004-05CenterAnnualReport.pdf>. [Consultado: el 24 de mayo de 2008]. 
Valles, Miguel. Técnicas cualitativas de investigación social. Madrid: Editorial Síntesis, 2000.

Vangen, Siv y Huxham, Chris. "The Tangled Web: Unraveling the principle of Common Goals in Collaborations", Journal of Public Administration Research and Theory 22/4 (2011): 731-760.

Wohlstetter, Priscila; Smith, Johana y Malloy, Courtney. "Strategic Alliances in Action: Toward a Theory of Evolution", The Policy Studies Journal 33 (2005): 419-442.

Wyner Walter y Samu, Sridhar. Nonprofit \& Business Sector Collaboration. New York: Haworth Press, 2003. 